\title{
Membrane Protein Mobility and Orientation Preserved in Supported Bilayers Created Directly from Cell Plasma Membrane Blebs
}

\author{
Mark J. Richards ${ }^{1}$, Chih-Yun Hsia ${ }^{1}$, Rohit R. Singh ${ }^{1}$, Huma Haider ${ }^{1}$, Julia Kumpf ${ }^{2}$, Toshimitsu \\ Kawate $^{2}$, Susan Daniel ${ }^{1^{*}}$ \\ ${ }^{1}$ School of Chemical and Biomolecular Engineering, Cornell University, Ithaca, NY. \\ ${ }^{2}$ Department of Molecular Medicine, Cornell University, Ithaca, NY.
}

\section{SUPPORTING INFORMATION}

Contained herein are supporting information and supplemental video files organized in the following sections:

1. Images of cell expression of P2X2-Neon and YFP-GPI

2. Details on our tracking algorithm

3. Comparison of single molecule tracking with FRAP

4. FRAP recovery curve of R18 in a bleb bilayer

5. Bleb size and concentration measurements

6. Bleb batch variability assessed by quartz crystal microbalance with dissipation

7. Sample images showing orientation assay results

8. Thrombin activity controls

9. Binding of antibodies to endogenous protein in the bleb bilayer

10. Sample movies demonstrating mobility of membrane proteins in bleb-supported lipid bilayers. 
1. Expression of P2X2-Neon on GPI-YFP in HeLa cells. Cells transfected with either GPIYFP or P2X2-Neon plasmids were grown and imaged under brightfield and fluorescence microscopy to evaluate expression (Figure S1).
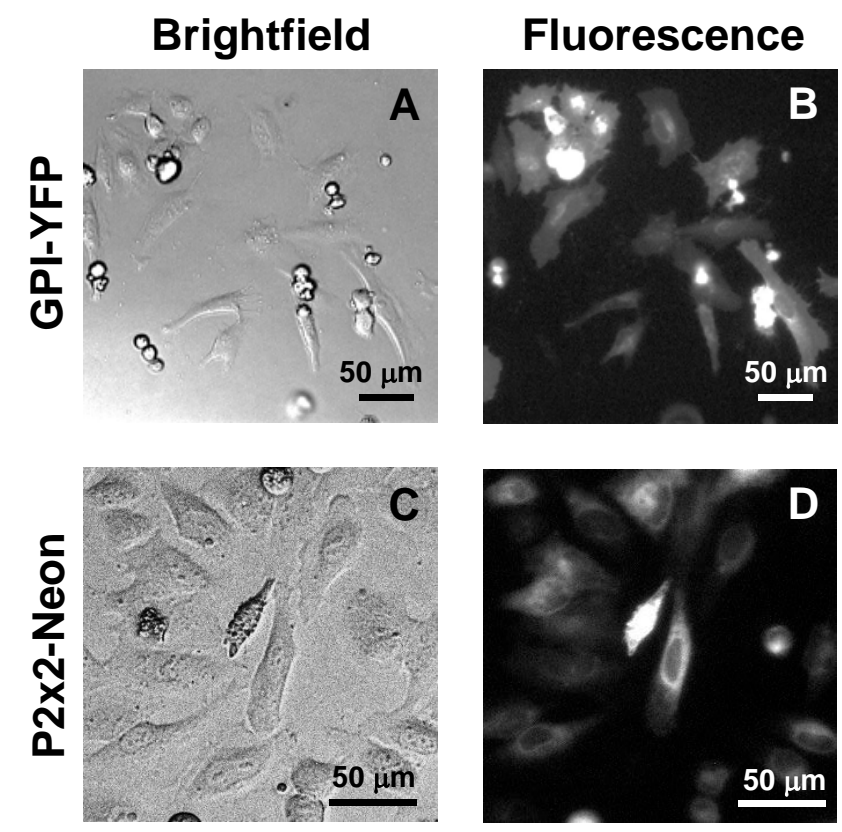

Figure S1. Expression of GPI-YFP (A,B) and P2X2-Neon (C,D) in HeLa cells in $10 \mathrm{~cm}$ dishes. Brightfield contrast images and corresponding fluorescent images of HeLa cells are shown.

2. Tracking algorithm and trajectory analysis. Linking of trajectories was performed by finding maximizing value of $w_{t}=w_{i} f_{i}+w_{\Delta i} f_{\Delta i}+w_{d} f_{d}$ (S1) among all candidate particles in each frame ${ }^{1}$. Weighting values used were: $w_{i}=0.05, w_{\Delta i}=0.05, w_{d}=0.9$. Equations for each criteria are given as:

$$
\begin{gathered}
f_{i}=e^{\left(\frac{I-\langle I\rangle}{\langle I\rangle-I_{b g}}\right)} \\
f_{\Delta i}=e^{\left(\frac{-\Delta I^{2}}{2 \sigma_{\Delta I}^{2}}\right)} \\
f_{d}=e^{\left(\frac{-d^{2}}{2 \sigma_{d}^{2}}\right)}
\end{gathered}
$$


In these equations, $I$ represents the intensity of the particle, $I_{b g}$ is the background intensity averaged over all frames, $\Delta I$ is the change in intensity from the previous frame and $d$ is the displacement from the previous frame. Particle candidates are only accepted into trajectories if they have $w_{t}>0.7$.

Moment scaling spectrum analysis is performed on each trajectory $j$ individually. Let $M$ be the length of the trajectory, $n$ be the frame number, $\Delta n$ be the frame step displacement (in this paper, we limited $\Delta n$ to $M / 4$ to limit error ${ }^{2}$ ), $x$ be the position of the particle, $p$ be the moment order, and $\|\cdot\|_{2}$ be the Euclidean norm. First, the displacement moments $\mu$ are calculated as

$$
\mu_{p}=\frac{1}{M_{j}-\Delta n} \sum_{n=0}^{M_{j}-\Delta n-1}\left\|x_{j}(n+\Delta n)-x_{j}(n)\right\|_{2}^{p}
$$

for $p$ values from 1 through 6 . Each moment follows a power law where $\mu_{p} \alpha \delta t^{\gamma(p)}$. The exponential factors, $\gamma(p)$, are determined by a linear regression through $\log \left(\mu_{p}\right)$ vs $\log (\delta t)$. If the diffusion process is strongly self-similar, the moment scaling spectrum, i.e. $\gamma(p)$ vs. $p$, will be linear with a slope defined as $\beta$ which quantifies the type of diffusion. Species with $\beta$ values near 0.5 are exhibiting normal diffusion, $0.5<\beta<1$ are superdiffusive and $0<\beta<0.5$ are subdiffusive.

In this paper, reported diffusion coefficients are the initial diffusion determined from the slope of the mean squared displacement vs. time lag plot (Eq. S4) up to $2 \delta t$. These are referred to elsewhere as $D_{0}$ or $D_{1-3}$ and are represented as the diffusion of a particle independent of the diffusion mode.

$$
M S D=4 D \delta t
$$

\section{Comparison of single molecule tracking with FRAP using streptavidin-biotin tracking in}

artificial bilayers. As an additional check on our tracking methods, we compared the single molecule tracking results with ensemble FRAP measurements for diffusivity of fluorescent 
Alexa Fluor 594 Streptavidin (ThermoFisher) bound to biotin-X-DHPE (ThermoFisher) in our standard POPC-PEG5k cushioned bilayer. The use of these probes enabled tuning of bilayer concentration to both the low range for SPT and the high range required for FRAP. A FRAP experiment was performed using the POPC-PEG5k lipids doped with $0.2 \%$ biotin-X-DHPE. After bilayer formation, $1 \mu \mathrm{L}$ of $2.5 \times 10^{-5} \mathrm{mg} / \mathrm{mL}$ streptavidin-594 was added, allowed to bind for 10 minutes and then rinsed. The diffusivity reported from three FRAP experiments was 0.98 $\pm 0.15 \mu \mathrm{m}^{2} / \mathrm{s}$, in the expected range for a probe of this type in a highly fluid bilayer. The mobile fraction was determined to be $0.96 \pm 0.05$.

Next, we generated a single particle tracking sample using POPC-PEG5k doped with $10^{-6} \%$ biotin-X-DHPE. After bilayer formation, $30 \mu \mathrm{L}$ of $0.1 \mathrm{mg} / \mathrm{mL}$ bovine serum albumin (BSA) was added to prevent nonspecific binding of streptavidin and rinsed after 10 minutes. Then, $1 \mu \mathrm{L}$ of $2.5 \times 10^{-5} \mathrm{mg} / \mathrm{mL}$ streptavidin-594 was added, allowed to bind for 10 minutes and rinsed. The diffusivity measured from single particle tracking was $0.89 \pm 0.30 \mu \mathrm{m}^{2} / \mathrm{s}$, close to that of the FRAP results (Figure S2, A). To provide a second point of comparison to further compare these methods, we looked at using a lipid bilayer composition that would provide a lower diffusion coefficient. We used a mixture of 60\% N-palmitoyl-D-erythro-sphingosylphosphorylcholine (PSM) and 40\% ovine wool cholesterol (both from Avanti Lipids) that generates a liquid ordered phase and doped in 10-6 \% biotin-X-DHPE. From the SPT experiments on the ordered phase bilayer, streptavidin diffused with an average diffusivity of $0.58 \pm 0.27 \mu \mathrm{m}^{2} / \mathrm{s}$ which is slower as expected for an ordered phase bilayer (Figure S2, B). Because the diffusion was determined from the initial slope of the MSD, these values represent the free microdiffusion of the tracers, and as such may not correspond with the macro measurement from $\mathrm{FRAP}^{3,4}$.

Diffusional mode analysis shows that $43 \%$ of streptavidin trajectories in the disordered 
bilayer exhibited free diffusion with $0.4<\beta<0.6$, while only $15 \%$ of streptavidin trajectories in the ordered bilayer showed free diffusion. In both cases, the majority of trajectories were confined (Fig S2 C,D). The very high percentage of trajectories showing confined behavior in the ordered bilayer is expected because the increased lipid order and packing prevent free diffusion of membrane species. It is unclear why the disordered bilayer shows a significant degree of confinement in SPT tracking, but nearly full recovery in FRAP. We posit that it arises from the differences in the length and timescales of the experiments, whereby the SPT may be more sensitive to microdefects in the bilayer that hinder diffusion on the nanoscale length and millisecond time scale of these experiments.
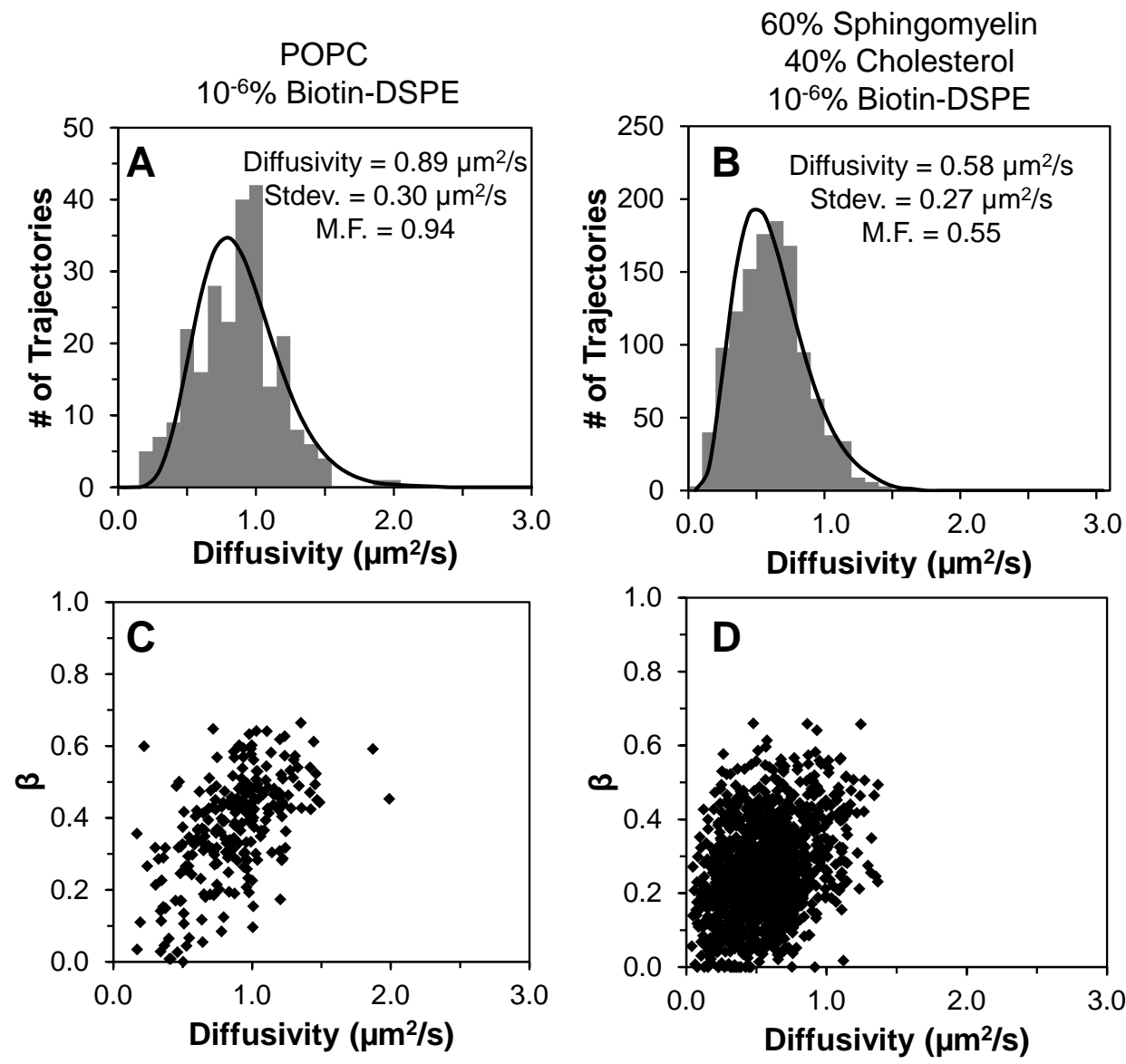

Figure S2. Diffusion coefficient histograms for Streptavidin-594 tracking experiments in disordered and ordered bilayers. Histograms are shown by gray bars and probability density function fits to a gamma distribution are shown with the black line $(\mathrm{A}, \mathrm{B})$. Additionally, scatter plots for each trajectory show the individual $\beta$-parameters and diffusivities (C,D). 


\section{Characterization of diffusion in bleb bilayer by fluorescence recovery after}

photobleaching (FRAP). To measure the diffusion coefficient of R18 in the supported bilayer, a $20 \mu \mathrm{m}$ diameter spot in the bilayer was bleached with a $4.7 \mathrm{~mW}$ wavelength tunable Argon/Krypton laser (CVI Melles Griot, Model 643-AP-A01) for 200 ms. The recovery of the fluorescence intensity of the photobleached spot was recorded for 9 minutes. Background was subtracted from each image to normalize for lighting variance. The recovery data was fit using a Bessel function following the method of Soumpasis ${ }^{5}$. The diffusion coefficient, $D$, was then calculated using the equation: $D=\frac{w^{2}}{4 t_{1 / 2}}$, where $w$ is the width at half-maximum of the Gaussian profile of the focused beam and $t_{1 / 2}$ is the recovery half-time. A sample recovery curve is shown in Figure S3.

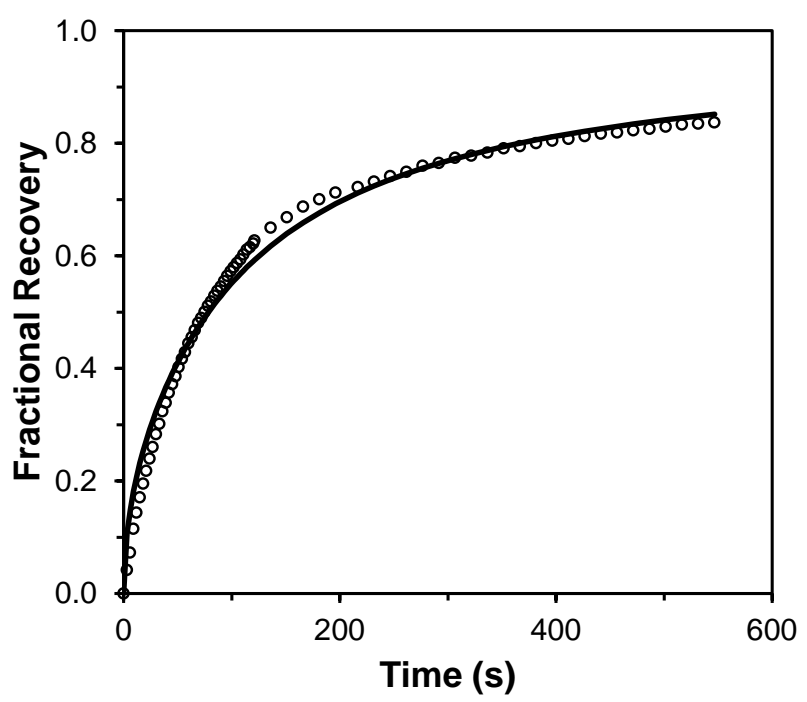

Figure S3. Example FRAP of R18 molecules in bleb bilayer (blebs + POPC-PEG5k). A small circle of R18 molecules was bleached with a laser at $0 \mathrm{~s}$. The fluorescence intensity recovers over time as the R18 molecules diffuse freely in the bilayer indicating that the blebs have fused into the SLB. Fitting of experimental data (diamonds) based on Soumpasis' equation is shown by the solid line. $\mathrm{D}=0.3 \mu \mathrm{m}^{2} / \mathrm{s}$.

5. Bleb size and concentration measurements. A ZetaSizerNano (Malvern) was used to measure particle sizes for undiluted samples of harvested blebs. Settings were chosen to maximize particles in focus with in-range signal intensities. Duplicate runs were performed on 
each sample and each sample showed similar results. The induced blebbing cases were about twice the concentration of the native samples (Figure S4). This factor may be partly attributed to variations in viability and cell density, but in all cases, cells were grown to similar confluency before inducing blebbing. Both conditions had two main peaks, one around $100 \mathrm{~nm}$ and a second around $200 \mathrm{~nm}$. The $200 \mathrm{~nm}$ peak represented a larger portion of the bleb population in the induced case than it did in the native, chemical-free blebbing case. There seems to be more dispersity in size in the induced case, maybe indicating that the process has some variability in terms of how it acts on certain regions of the membrane or based on local chemical concentrations.
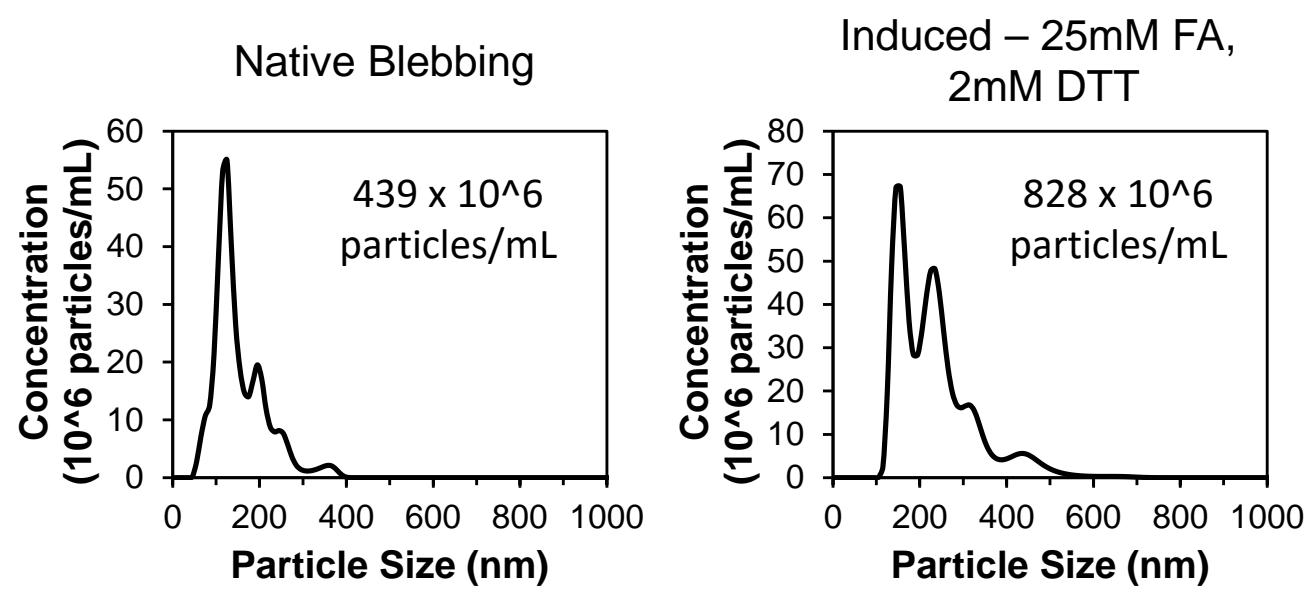

Figure S4. Particle size profiles for blebs generated using the native, chemical free blebbing (left) and chemically induced (right) methods. Total particle concentrations are given in insets.

6. Quartz crystal microbalance with dissipation (QCM-D) batch variability. To see how bleb surface coverage varies from batch to batch when using the same blebbing conditions, QCM-D was used to monitor bleb adsorption of three different batches. For each bleb batch, three (or more) independent experiments were performed on different days. Frequency and dissipation signals (Fig. S5) show that bleb surface coverage is reasonably similar across batches; this result suggests that bleb concentration across batches is fairly consistent. 
To be sure that bleb adsorption kinetics are solution concentration dependent, we also performed QCM-D experiments using blebs with different dilutions. Fig S6 shows that the surface coverage is limited by solution concentration, and hence we can conclude that the reproducibility shown in Fig S5 is due to the uniformity in bleb concentration.

(a)

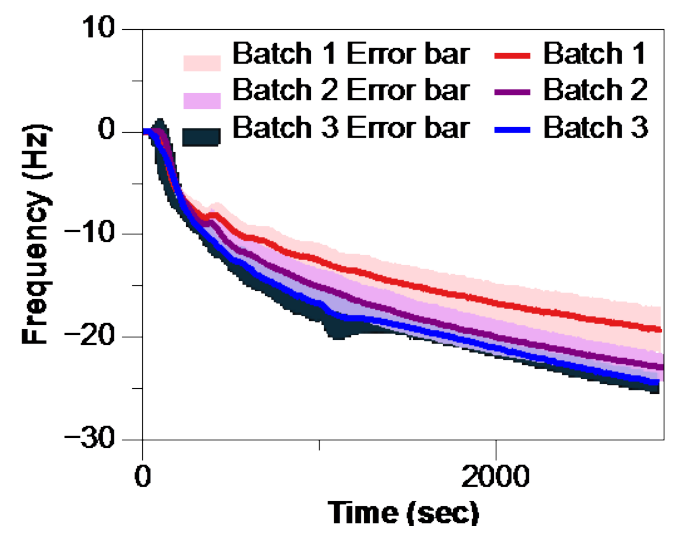

(b)

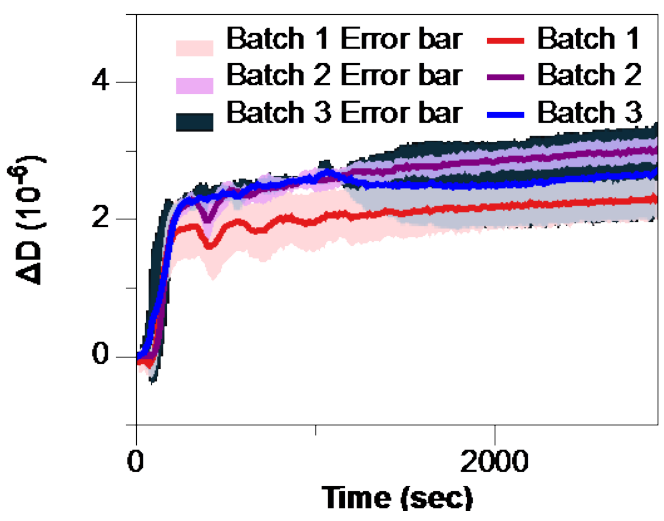

Figure S5. The (a) frequency and (b) dissipation signals showing the adsorption of three different batches of blebs. Each batch of blebs was used to perform three (or more) different independent experiments.

(a)

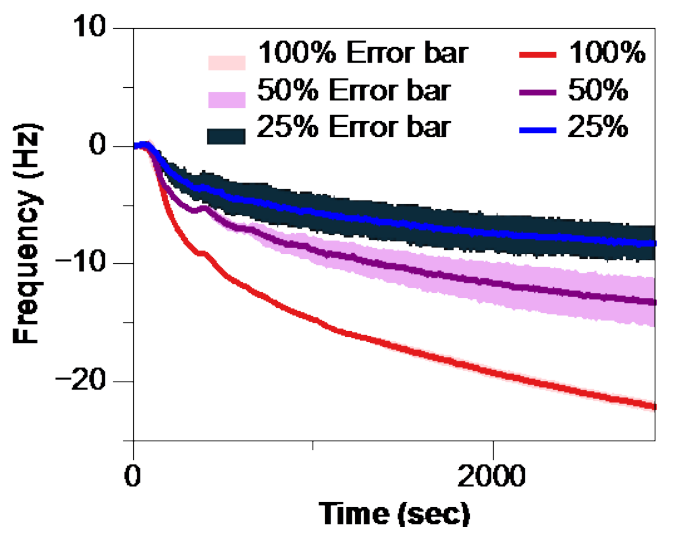

(b)

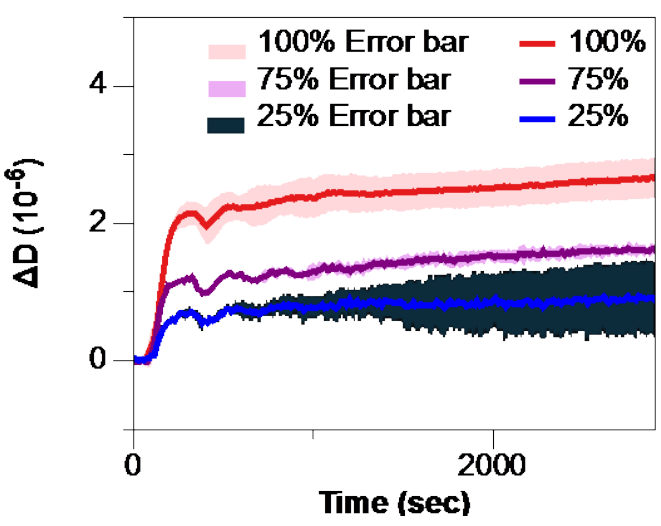

Figure S6. The (a) frequency and (b) dissipation signals showing the adsorption of bleb solution with different concentration. 100\%: bleb solution without dilution; 50\%: 50: 50 bleb solution to PBS in volume; 25\%: 25: 75 bleb solution to PBS in volume. Each concentration of bleb solution was used to perform three (or more) different independent experiments.

7. Orientation assay for proteins in blebs and SLBs. To determine protein orientation, enzymatic accessibility assays were performed on either in-tact blebs or bleb-based SLBs 
containing either GPI-YFP or P2X2-Neon. First a baseline image series was recorded of various locations on the sample surface, and then enzyme was added. Image series were recorded at 10, 20, 30 and 40 minutes after addition of enzyme. Figure S7 shows sample images from each time point for GPI-YFP, while Figure S8 shows sample images for P2X2-Neon. In the case of GPIYFP, for both blebs and bilayers, signal drops off rapidly after addition of proteinase K, while control samples without enzyme addition show a small amount of steady photobleaching. For P2X2-Neon, no apparent change is detected after addition of enzyme, and in both test and control cases only photobleaching is evident.

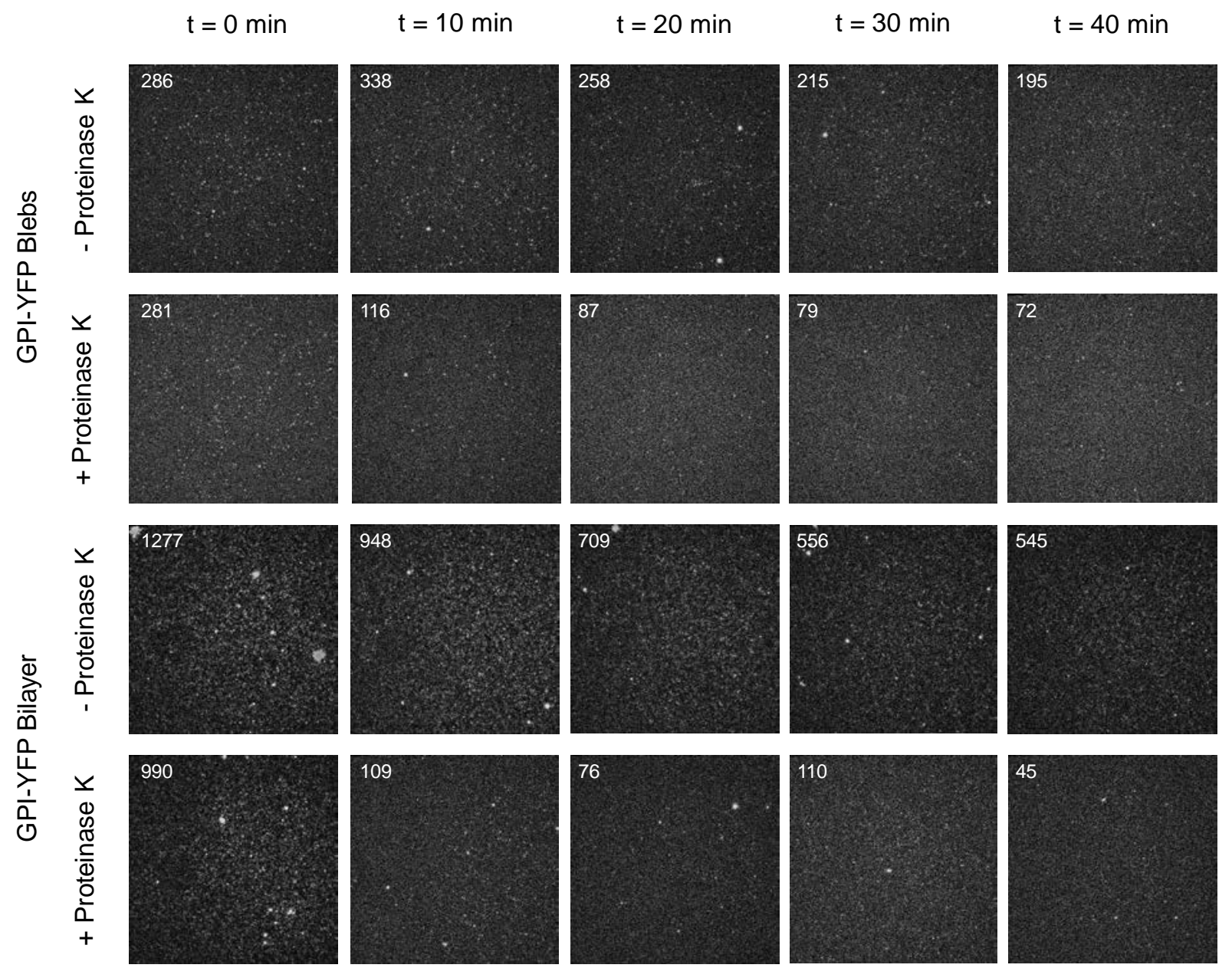

Figure S7. GPI-YFP protein image series in blebs and bilayers with and without addition of proteinase k enzyme. Representative images are shown for each time point: before addition of enzyme, 10, 20, 30 and 40 min after addition. Average particle counts for the time point are shown as insets in each image. In the bleb and bilayer cases, a large drop in particle count is observed after addition of proteinase k. Images are 82 x $82 \mu \mathrm{m}$. 


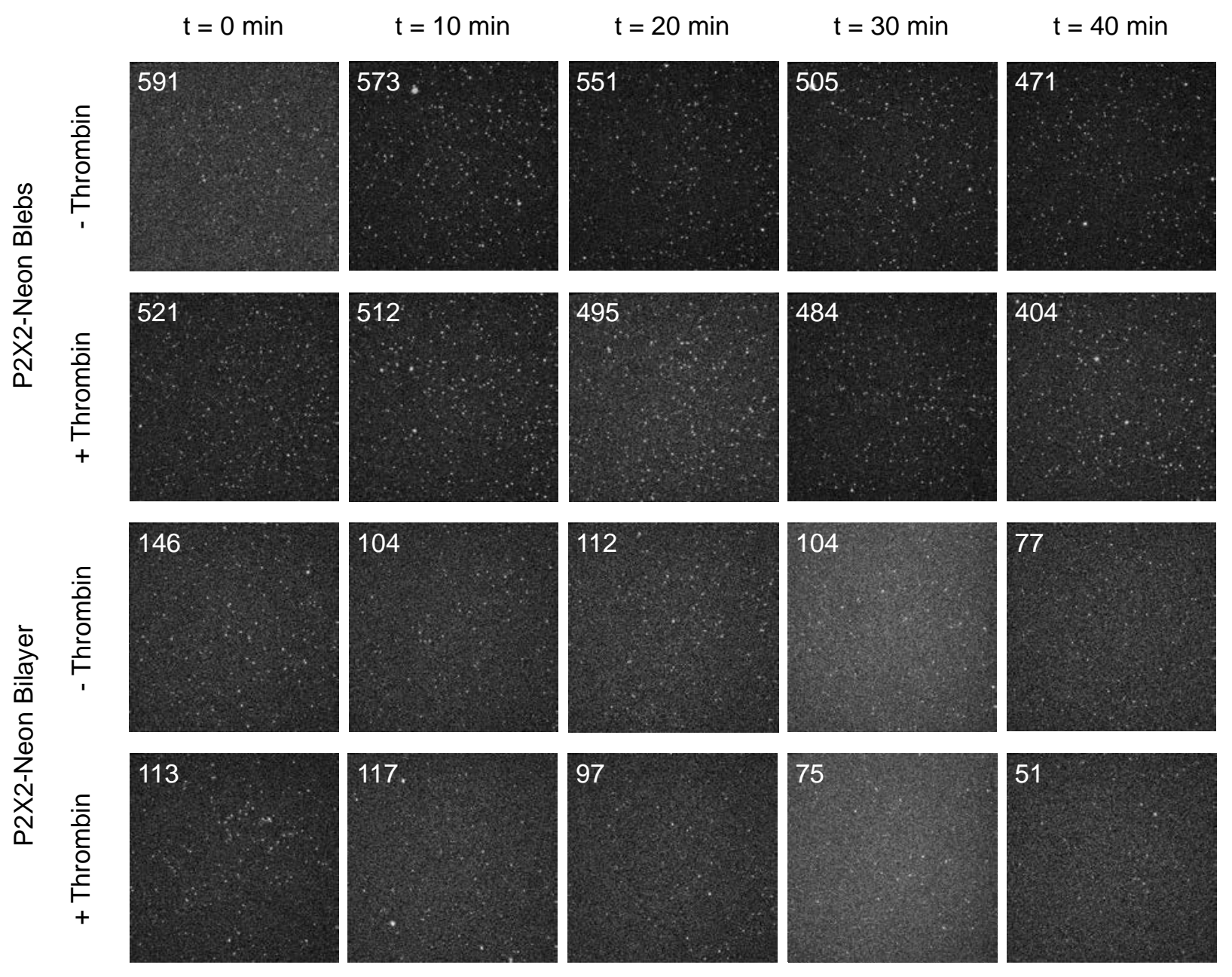

Figure S8. P2X2-Neon receptor image series in blebs and bilayers with and without addition of thrombin enzyme. Representative images are shown for each time point: before addition of enzyme, 10, 20, 30 and 40 min after addition. Average particle counts for the time point are shown as insets in each image. No difference was observed between enzyme and control experiments. Images are 82 x $82 \mu \mathrm{m}$.

8. Orientation assay results on scrambled blebs containing P2X2. To verify activity of thrombin on the P2X2-Neon protein constructs used in this work, we carried out a control experiment where blebs were initially scrambled before formation of the supported lipid bilayer. To do this we followed the general approach of bleb sonication devised in Pace et $\mathrm{al}^{6}$. In our experiments, P2X2-Neon containing blebs were mixed with POPC-0.5\%PEG5K vesicles and then subjected to both tip sonication using a Branson Sonifier 450 for $1 \mathrm{~min}$ at 30\% level 3 and bath sonication for $1 \mathrm{hr}$. Bilayers were formed in wells using this solution. Next, thrombin was 
added to one well to cleave at the thrombin cleavage site between the P2X2 protein and the neon fusion protein. Images at 100x magnification were taken and particle counts at time 0 and 60 minutes were tallied. Figure S9 shows that in the presence of thrombin, the mixed bilayer dropped in particle count, thus proving that the thrombin is in fact capable of cleaving out P2X2 protein when the site is exposed to it.

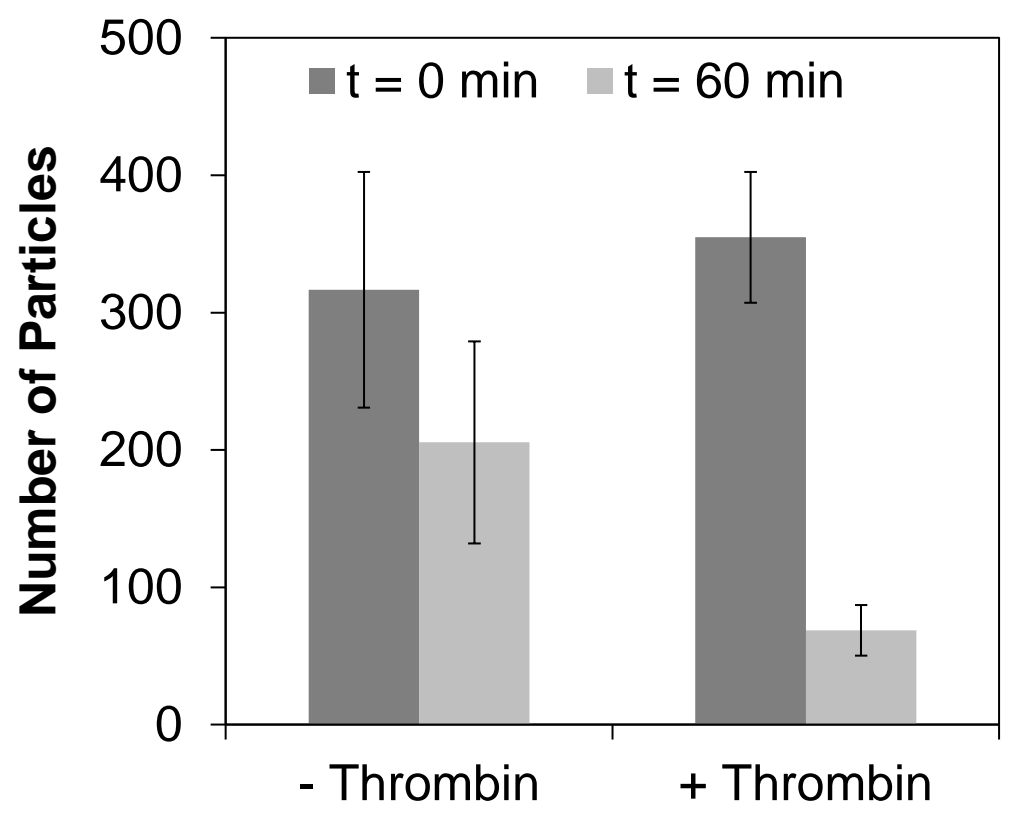

Figure S9. Comparison of particle counts without (-) and with (+) thrombin for scrambled P2X2 bilayers exposing inner side of the P2X2 to the bulk phase. After 60 minutes with thrombin, a significant number of particles were lost relative to the (-) case, which represents the amount of photobleaching. Error bars represent the standard deviation for 5 different bilayer locations.

9. Antibody binding to endogenous proteins on bleb SLB surface. To confirm that the PEGylated bilayer does not inhibit binding of extracellular species to receptors on the bleb bilayer, we examined the ability of transferrin receptor to bind its antibody. Transferrin is natively expressed in the HeLa cell used here to form the blebs. A FITC-labeled monoclonal antibody against human transferrin receptor CD71 (clone MEM-75, ThermoFisher Scientific) was used. A blebbed SLB prepared with POPC-PEG5k lipids was made as previously described, 
along with a POPC-PEG5K bilayer (no blebs) as a control case. After blocking with $0.005 \mathrm{mg} / \mathrm{ml}$ bovine serum albumin (BSA) for 150 minutes to reduce non-specific adsorption, the samples were thoroughly rinsed with PBS. Next, $1 \mu$ l of the antibody solution was added to each well and incubated for 20 minutes before thoroughly rinsing with PBS to remove unbound antibodies. Fluorescent images of the bilayer using a 100x objective were recorded and the fluorescent particles were counted from these images. These results, shown in Figure S10, clearly show that the antibody is able to bind its target in the blebbed SLB despite the presence of PEG. The control bilayer showed limited non-specific adsorption. Additionally, this result provides further support for the bleb bilayer orientation we concluded from the thrombin enzyme experiments because the antibody binds to the extracellular domains of transferrin receptor.
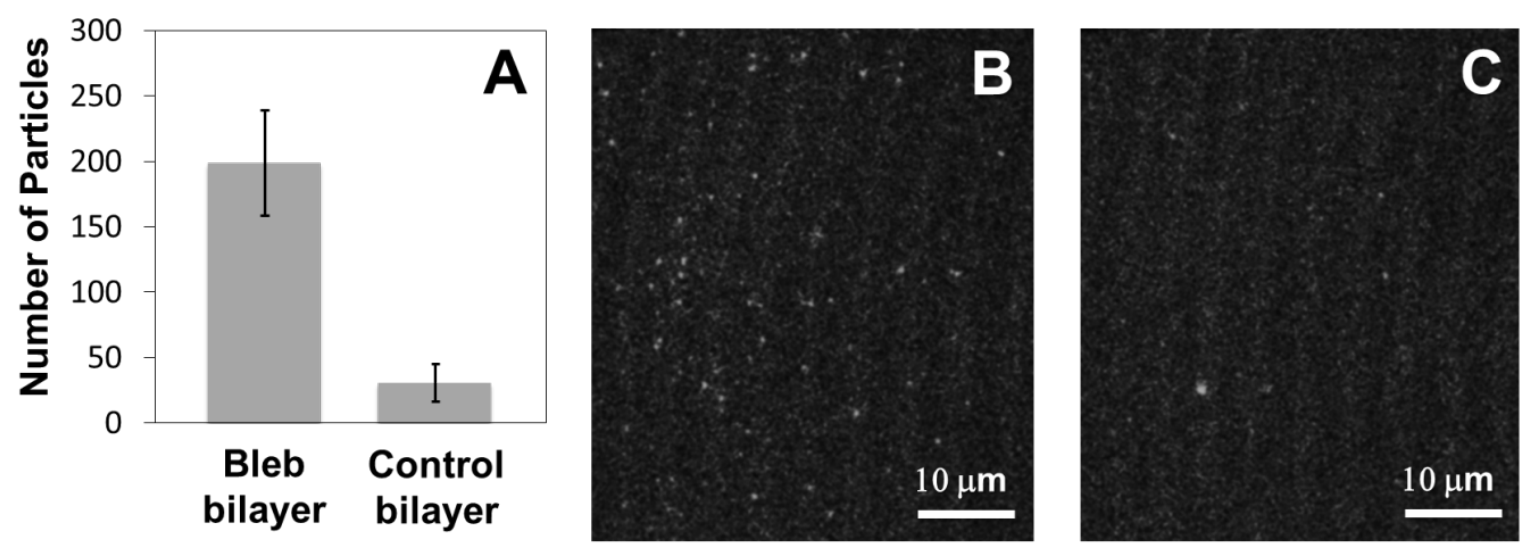

Figure S10. Binding of FITC-labeled anti-CD 71 antibody to blebbed SLB. The number of fluorescent particles, averaged over at least seven images, is shown in (a) for a blebbed SLB and a non-blebbed SLB. Representative images are shown for (b) a bleb SLB, and (c) a control SLB.

\section{Supplemental Video Attachments}

V1. TIRF image series showing rupture of GPI-YFP blebs during addition of POPC-PEG5k vesicles. Initially, GPI-YFP proteins are confined to the adsorbed blebs, some in large clusters, but as fusogenic lipid vesicles are added, it is clear that the proteins are able to spread out from blebs and diffuse laterally in the newly forming bilayer. This early-time diffusion shortly after addition of lipids is more hindered than the diffusion we report on after waiting for 30 min to allow complete bilayer formation. The field of view is $82 \times 82$ microns and playback is in real time. - "Adding POPCPEG5k to GPI-YFP on glass" 
V2. TIRF image series showing rupture of P2X2-Neon blebs during addition of POPC-PEG5k vesicles. Initially, P2X2-Neon proteins are visible in isolated blebs that are adsorbed to the surface. As fusogenic lipid vesicles are added, diffusion out from these positions is apparent, indicating bleb rupture into the lipid bilayer. The field of view is 82 x 82 microns and playback is in real time. - “Adding POPCPEG5k to P2X2-Neon on glass”

V3. TIRF image series of P2X2-Neon proteins diffusing in the SLB formed from blebs and POPC-PEG5k vesicles. Colored tracks are superimposed on the image to highlight some of the particle trajectories determined by the tracking algorithm. The field of view is $82 \times 82$ microns and the playback is in real time. - P2X2-Neon Tracking in POPCPEG5k bilayer

\section{References}

(1) Smith, M. B.; Karatekin, E.; Gohlke, A.; Mizuno, H.; Watanabe, N.; Vavylonis, D. Interactive, ComputerAssisted Tracking of Speckle Trajectories in Fluorescence Microscopy: Application to Actin Polymerization and Membrane Fusion. Biophys. J. 2011, 101, 1794-1804.

(2) Sbalzarini, I. F.; Koumoutsakos, P. Feature Point Tracking and Trajectory Analysis for Video Imaging in Cell Biology. J. Struct. Biol. 2005, 151, 182-195.

(3) Sonnleitner, A.; Schütz, G. J.; Schmidt, T. Free Brownian Motion of Individual Lipid Molecules in Biomembranes. Biophys. J. 1999, 77, 2638-2642.

(4) Saxton, M. Single-Particle Tracking: The Distribution of Diffusion Coefficients. Biophys. J. $1997,72$.

(5) Soumpasis, D. M. Theoretical Analysis of Fluorescence Photobleaching Recovery Experiments. Biophys. J. 1983, 41, 95-97.

(6) Pace, H. P.; Simonsson Nyström, L.; Gunnarsson, A.; Eck, E.; Monson, C. F.; Geschwindner, S.; Snijder, A.; Höök, F. Preserved Transmembrane Protein Mobility in Polymer-Supported Lipid Bilayers Derived from Cell Membranes. Anal. Chem. 2015, DOI: 10.1021/acs.analchem.5b01449. 